\title{
Digital Humanities for Academic and Curatorial Practice
}

\author{
ANGELICA FEDERICI, University of Cambridge, UK \\ JOSEPH WILLIAMS, Duke University, NC, USA
}

\begin{abstract}
The Digital Humanities have challenged all disciplines of Art History to engage with new interdisciplinary methodologies, learn new tools, and reevaluate their role within academia. In consequence, art historians occupy a new position in relation to the object of study. Museums have been equally transformed. The possibilities of creating virtual realities for lost/inaccessible monuments poses a new relationship between viewer and object in gallery spaces. Digital Humanities interventions in museums even allow us to preserve the memory of endangered global heritage sites that cease to exist or are inaccessible (celebrated examples including the lost Great Arch of Palmyra reconstructed with a 3D printer). Curatorial practices are now trending towards a sensorial and experiential approach. Is the role of Digital Humanities, in academic as well in museum settings, to "reveal" the object itself, through an empirical display of existing material, or to "reconstruct" something of the original experience of the object to engage spectators? Can we propose a reconciliation between these two "poles"? The Sixth International Day of Doctoral Studies promoted by RAHN aims to investigate the role of Digital Humanities by fostering a dialogue between the protection of cultural heritage sites, museology, the history of art, and the digitalization of Big Data.
\end{abstract}

Key words:

Digital Humanities, Conference Proceedings, Revealing, Reconstructing

SDH Reference: Angelica Federici and Joseph Williams 2019. Digital Humanities for Academic and Curatorial Practice. SDH, 3, 2, 117-121.

DOI: $10.14434 /$ sdh.v3i2.27718

\section{INTRODUCTION TO THE CONFERENCE PROCEEDINGS “DIGITAL HUMANITIES FOR ACADEMIC AND CURATORIAL PRACTICE"}

The Digital Humanities have challenged all aspects of the discipline of Art History to engage with interdisciplinary methodologies, innovative technologies, and ground-breaking approaches to pedagogy. By combining new and established tools of scholarly enquiry including but not limited to archival research, empirical display of extant objects, meticulous study of building structures with photogrammetry/point cloud, building information modelling, digital cataloguing, and virtual reality, this conference has furthered in exciting new ways existing scholarship on Digital Humanities. This

Author's address: Angelica Federici, The Department of History of Art, 1-5 Scroope Terrace, Cambridge, CB2 1PX, UK; email: angelica.federici@uniroma3.it; Joseph Williams, Wired! Lab for Digital Art History \& Visual Culture, Smith Warehouse, Bay 11, Duke University, Durham, NC, 27708, USA; email: j.chandler.williams@gmail.com

(C) [2018] by the authors; licensee Studies in Digital Heritage, IU, Bloomington (IN), USA. This article is an open access article distributed under the terms and conditions of the Creative Commons Attribution License (CC BY-NC). 


\section{3:118 A. Federici and J. Williams}

interdisciplinary meeting has set new agendas for research, challenged traditional methodologies and contributed significantly to the multifaceted nature of this discipline.

The advances in this field were showcased through a variety of projects which were broadly separated into two subcategories. Whether through revealing or reconstructing we believe Digital Humanities need to further empirically our knowledge on a particular subject matter. Is the digital element ultimately a means by which the Humanities are revealed or reconstructed? The discipline is broadly defined as an intersection or application of computational tools and methods to traditional humanities.

The regulatory thematized effects of revelation broaden our delineation of the subject. Indeed, by revealing Digital Humanities are not exclusively tied to the study of texts by which a hermeneutical stance makes us understand new and hidden signifiers. While some of these projects do consider language as a principal medium of investigation, others reveal patterns and hidden meanings by constructing databases that aim to organize material systematically. For example, The Faculdade de Belas-Artes da Universidade de Lisboa (FBAUL) holds a Collection of Lithographs, dated between 1840 and 1911, related to the memory of teaching Drawing in the nineteenth century. The FBAUL Virtual Print Room is a bilingual (Portuguese/English) website project executed by Alberto Faria for his dissertation, that enables users to access the Inventory and Knowledge of the Collection, in order to build connections with other Archives around the world using GIS.

The creation of databases also draws us into the Big Data question in so much as large data sets may be analyzed computationally to reveal patterns, trends, and associations, especially relating to human behavior and interactions. As part of her dissertation project Giuditta Cirnigliaro conducted a comparative analysis of Leonardo's folios featuring fables, emblems, and engineering projects, identifying the archetypes of this interaction in the books contained in his personal library to examine the convergence of his use of empirical, diagrammatic, and pictorial strategies toward the investigation of nature. The identified patterns were subsequently catalogued and examined through the web-publishing platform "LILeo" created in collaboration with the Rutgers Digital Humanities Laboratory uncovering the origins of his interdisciplinary research. Large databases also raise questions of copyright especially in so far as information is now largely open access and intellectual property is infringed. This problematic aspect was taken into consideration by Dario Haux a specialist in legal policy frameworks who analyzed this theme in a global context in relation to IT and intellectual property rights law, with a paper "Sul paradigma della preservazione del nostro patrimonio globale con l'informatica digitale."

In the context of Digital Humanities we learned that Revealing is a homonym, a polysemy characterized by multiple complementary signifiers. Ji Young Park explored the quality of this verb by analyzing it from a communications perspective, specifically the media discourses of South Korean national museums with a presentation on Digital provenance research for Asian art: revealing an object's past, reconstructing its discourse. To a certain extent one may argue that the Digital Humanities are disclosing evidence by new creating cognitive pathways and new behavioral patterns towards knowledge.

Aside from the theme of "revealing," that of the "reconstruction" of the primary experience of objects loomed large in the conference. By now digital reconstructions of historical spaces and objects have 
advanced well into the realm of lifelike experiences. The Virtual Reality elements of the new tour at the Domus Aurea in Rome prove that these technologies create a more immediate impression of a site than do standard texts and diagrams. However, scholars and curators share concern that these models sacrifice rigor for impact. The old dichotomy is not between digital modes of representation and traditional ones, but between restoring an expressive, "original" experience of an object and presenting the artifact in its current state--in other words, reconstructing vs. revealing. Over a long period of time, the former approach has come to be perceived as conjectural and popular, the latter as rigorous and scholarly.

But digital revolutions in modelling, VR (Virtual Reality), 3-D printing, and interactive content have not simply produced more convincing simulacra. They have also begun to bridge the old dichotomy between reconstructing and revealing. These technologies create interactive experiences in which users can sift through layers of primary data and the theories they support. Museums and scholarly websites create such layers in different ways, from traditional drop-down menus and tick-boxes to augmented reality apps. Interactive layers invite users to engage simultaneously with a lifelike experience and with its supporting scholarly apparatus, revealing the connective tissue between evidence and hypothesis. In addition, this interactive content takes advantage of current scholarly interest in phenomenology, the methodical interpretation of primary experience. In a phenomenological lens, a user's experience is key data.

BYZART, an online database of Byzantine art and archaeology, gives users a privileged opportunity to interact with scholarly content. As Giulia Marsili and Lucia Maria Orlandi discuss, BYZART, which exists on the Europeana platform, presents a broad audience with a large variety of media representing Byzantine art and historiography. The great breadth of the collection, spanning archives that cover the entire Byzantine Empire (many of them hitherto unpublished), depends on multiple levels of scholarly and popular interaction. In addition to the core team members, who curate the website, and the general public, who may peruse the site but cannot contribute content, is a third category: data providers, who share access to an Omeka-based platform for submitting new content for review. As a result, the site is built up through reciprocal interaction between the providers and the core team over time. The former constantly expand the types of content, while the latter adjust the browse options to pave inroads through the shifting material. The content and its structure are both subject to constant change. Users thus become an audience to the unfolding scholarly discourse on how to systematize the diversity of Byzantine art and art history.

For example, the effort to incorporate the archive of Giuseppe Bovini into BYZART posed fruitful new challenges of classification and digital searching. The archive is full of the eminent art historian's personal notes and photographs, many documenting new excavations in the 1960s. The BYZART team has chosen to catalog all this information, which illustrates Bovini's scholarly personality and the development of his research method. In addition, these materials reveal how Bovini interacted with objects and sites in an earlier, more robust state of preservation. Making this material available for the first time can give BYZART users a vicarious experience of artistic heritage prior to its deterioration. The complexity of the Bovini archive opens several different axes of historical as well as historiographical inquiry. BYZART relies on such challenges to continue sharpening its interactive platform. 


\section{3:120 A. Federici and J. Williams}

Like BYZART, the Virtual Photo Library Project hosted by D.I.R.A.A.S. (Dipartimento di Italianistica, Romanistica, Antichistica, arti e spettacolo) at the University of Genoa employs a collective access model for uploading content and drawing new connections within it. As Martina Massarente explains, the concept of the online photo library is to allow users--both specialists and nonspecialists alike--to access digitized photographic materials from various repositories in order to understand not only the objects themselves, but the patterns and links among them. In particular, Massarente is interested in what these photographs may reveal about the evolution of art historical pedagogy--part of the collection, for example, makes use of the photographic materials of Giusta Nicco Fasola, Genoa's first professor of art history. Through the interaction of Wordpress and the Collective Access platform, users are invited to contribute content themselves, but they are guided through the data entry by a set of prompts, drop-down menus, and pre-populated fields. The interface adeptly subjects the efforts of non-specialist contributors to rigorous cataloging standards.

In addition to digitizing analog materials, more and more museums and web galleries are employing digital 3-D reconstructions. This poses new questions regarding the engagement of users. To what extent do photogrammetric and scan-based reconstructions, which have strong optical and haptic verisimilitude, approximate an experience of the original object? Jane-Heloise Nancarrow considers the possibility of an "uncanny valley" effect when the reconstruction of an object or space too closely resembles its original while remaining obviously unreal. Nancarrow discusses alternative approaches to the problem, one to enhance the model with post-processing effects that overcome some of the unsettling markers of the object's fakeness, and another to offer the model as a 'raw' file that the user may download and interpret as he or she chooses, adding a level of interactivity. In both examples, Nancarrow's research into the psychological and emotional effects of experiencing digital objects warns us not to draw a one-to-one correlation between the experience of a convincing reconstruction and that of an original object.

Examples from beyond the current volume can be cited for the expanding field of object reconstruction in real and digital environments. A recent intervention by Kelly McClinton at the Eskenazi Museum of Art (Indiana University) used VR to combine user interaction and vivid reproduction. A fragment of a Roman marble satyr in the collection was brought to life for visitors. By standing near the fragment and donning a VR headset, visitors could call up augmentations using a series of menus, which floated before their eyes in real space. Clicking through the menus produced written descriptions and images contextualizing the fragment. One image was a lifelike 3-D photogrammetric model of a comparable marble satyr in Rome's Capitoline Museums. The user could move around the model and inspect it alongside the fragment: a much-enhanced version of the sideby-side comparisons popularized by Heinrich Wölfflin. This exhibition allowed users to interact with layers of information as he or she wished. First, the contextual information about the satyr was only available by opening the app; in other words, it was possible to approach the object unfettered by augmented reality. Second, the drop-down menus allowed the user to sort through different kinds of accompanying information. Third, by offering a 3-D visual document of an existing comparandum, the app tapped into the user's imagination: now equipped with the robust primary data of the photogrammetric scan, the user could imagine what the Eskenazi satyr would have looked like whole. This approach is much more transparent than the traditional method of simply creating a 
speculative model reconstruction, which usually hides the evidence supporting the scholar's choices.

The fabricators of Torart use digital recording and modelling software to aid their creation of physical sculpture models, such as their famous replica of the Great Arch of Palmyra. The Palmyra arch, reproduced by carefully orchestrated machines to a fine level of detail, stands in metaphorically for the presence erased by Daesh in 2015. The replica was unveiled in various locations, including Trafalgar Square in London. Though it would be wrong to say that the original arch was "rescued," the work of Torart has given general public access to an experience approximating one that long remained the province of traveling scholars, and that is now entirely in the past.

Databases and virtual/physical reconstructions of heritage objects have used increasingly sophisticated technologies and user interfaces to elevate the experiential to the realm of the scholarly. Lifelikeness, interactivity, and transparency have become complementary goals. There is no longer the need to choose between reconstruction and revealing. Digital technologies support a happy marriage of both

Revealing patterns, meanings, and methodological opportunities in primary evidence, and reconstructing vivid experiences-are not opposed, but complimentary. On one hand, digital technologies can make humanistic interpretations more rigorous. The methodological intricacy of the Digital Humanities often entails the foundation of research labs, where research is specialized, collaborative, iterative, and transparent. In these ways, the Humanities can learn from the model of the Sciences. On the other hand, digital technologies make the Humanities more accessible, creating platforms that invite the audience "into the lab." Without sacrificing scholarly rigor, the Digital Humanities make academic discourse more available, appealing, and engaging to the greater public. This improves the long-term viability of Humanities fields, not to mention their access to resources.

Web databases and VR experiences can be dazzling, and digital humanists may be tempted to play the role of "connoisseur behind the curtain." But this need not be the rule. Digital technologies offer scholars the opportunity to demystify, as well as expand, their methods. Good Digital Humanities is a collective effort made of a team of people from different disciplines and has lot in terms of advancement in the field to learn from empirical analysis of material a practice which to this day is still more methodologically advanced in the sciences.

Received July 2019; revised November 2019; accepted December 2019

Studies in Digital Heritage, Vol. 3, No. 2, Publication date: December 2019 